УДК 616.151:612.392.61]-616.12-008.64-036.2

DOI:10.26435/UC.V0I4(29).227

\author{
Н.Т. Ватутин ${ }^{1,2}$, А.Н. Шевелёк ${ }^{1,2}$, А.Н. Загоруйко ${ }^{1}$ \\ ${ }^{1} Г 00$ ВПО «Донецкий национальный медицинский университет имени М. Горького», Донецк \\ ${ }^{2}$ Институт неотложной и восстановительной хирургии им. В.К. Гусака, Донецк
}

\title{
РАСПРОСТРАНЕННОСТЬ ДОГОСПИТАЛЬНОЙ И ГОСПИТАЛЬНОЙ ГИПОНАТРИЕМИИ У БОЛЬНЫХ С ДЕКОМПЕНСАЦИЕЙ ХРОНИЧЕСКОЙ СЕРДЕЧНОЙ НЕДОСТАТОЧНОСТИ
}

Хроническая сердечная недостаточность (ХCH) является одним из наиболее распространенных и прогностически неблагоприятных заболеваний сердечно-сосудистой системы, при этом полагают, что в ближайшие 30-40 лет следует ожидать увеличение её распространенности на 40-60\% [1]. Годовая смертность от ХСН достигает $6 \%$, а более 50 \% пациентов погибают в течение 4 лет после развития клинических симптомов заболевания [2].

Одним из факторов, определяющих клиническое течение и прогноз ХСН, являются нарушения водно-электролитного баланса, среди которых ведущее место занимает гипонатриемия [3]. Снижение уровня натрия крови является важным фактором риска неблагоприятного прогноза как при стабильном течении заболевания, так и при его декомпенсации [4]. Согласно результатам крупных исследований $[5,6,7]$, данное состояние ассоциируется с увеличением сроков пребывания в стационаре, частоты повторных госпитализаций и смертности.

По данным литературы [8], гипонатриемия является самой частой электролитной аномалией среди лиц, госпитализированных в стационар по различным причинам. У больных с ХCH её распространенность варьирует в пределах 18$27 \%$ и зависит от выборки пациентов. Однако нельзя исключить, что в реальной клинической практике этот показатель будет выше приведенных значений, поскольку, во-первых, исследование уровня электролитов крови, к сожалению, не является рутинной диагностической процедурой у больных ХCH, а во-вторых, даже выявленная по данным лабораторного исследования гипонатриемия часто остается без внимания со стороны лечащих врачей. Кроме того, нормальный уровень натрия крови при однократном измерении в первые дни госпитализации не исключает возможности дальнейшего его снижения на фоне активной диуретической терапии.
При этом частота прогрессирования догоспитальной гипонатриемии во время пребывания в стационаре также остается неизученной.

\section{ЦЕЛЬРАБОТЫ}

Целью нашего исследования стала оценка распространенности различных типов гипонатриемии у пациентов, госпитализированных по поводу декомпенсации ХCH.

\section{МАТЕРИАЛ И МЕТОДЫ}

Было проведено проспективное исследование с участием 396 пациентов, госпитализированных в Институт неотложной и восстановительной хирургии им. В.К. Гусака в связи с декомпенсацией ХСН за период 2013-2016 гг.

Критериями включения в исследование считали возраст старше 18 лет, госпитализацию в стационар по поводу декомпенсации ХСН и подписанное информированное согласие.

ХCH диагностировали в соответствии с современными рекомендациями. [9]. Критерием декомпенсации считали наличие как минимум двух из следующих признаков: одышка при незначительной физической нагрузке либо в покое, влажные хрипы в легких, периферические либо генерализованные отеки, пульсация яремных вен, рентгенологические признаки застоя по малому кругу кровообращения.

Критериями исключения были острый коронарный синдром, клапанные и септальные пороки сердца, миокардиты, гипертрофическая и дилатационная кардиомиопатии, другие состояния, ассоциированные с гипонатриемией (цирроз печени; ожоговая болезнь; острые расстройства желудочно-кишечного тракта; нефротический синдром, скорость клубочковой фильтрации < 15 мл/мин, надпочечниковая недоста-

( ) Н.Т. Ватутин, А.Н. Шевелёк, А.Н. Загоруйко, 2018

(c) Университетская Клиника, 2018 
точность, острая гиповолемия), заболевания, связанные с неадекватной продукцией антидиуретического гормона (опухоли легких, инфекционные процессы в легких, гипотиреоз), сопутствующая патология в стадии декомпенсации, беременность, онкологические заболевания, алкогольная и наркотическая зависимость, неспособность или нежелание соблюдать процедуры протокола исследования или подписать информированное согласие, участие в другом клиническом исследовании в течение предшествующих 30 дней.

У всех пациентов при поступлении в стационар и в течение госпитального периода лечения определяли уровень натрия в сыворотке крови. Исследование выполняли с помощью ионоселективных электродов на автоматическом биохимическом анализаторе «Cobas C 311» (Roche Diagnostics, Германия). Концентрацию электролита выражали в ммоль/л. С целью исключения случаев ложной гипонатриемии при наличии у больного выраженной гипергликемии производили перерасчет уровня натрия крови: полученное при лабораторном измерении значение увеличивали на 2 ммоль/л при повышении уровня глюкозы крови на каждые 5,6 ммоль/л выше верхней границы нормы [5, 10, 11].

Гипонатриемию диагностировали при снижении уровня натрия в сыворотки крови < 135 ммоль/л. Степень тяжести гипонатриемии оценивали в соответствии с общепринятыми рекомендациями [5]. При уровнях натрия 130-134 ммоль/л гипонатриемию расценивали как лёгкую, при 125-129 ммоль/л - как умеренную, и при < 125 ммоль/л - как тяжелую. Догоспитальной считали гипонатриемию, обнаруженную при поступлении, госпитальной - развившуюся на фоне лечения в условиях стационара. С целью оценки сроков развития госпитальной гипонатриемии исследование уровня электролитов проводили каждые 3 суток. У лиц с догоспитальной гипонатриемией также анализировали динамику уровня натрия во время госпитализации, при этом в случае снижения уровня натрия $\geqslant 3$ ммоль/л по сравнению с исходным значением диагностировали прогрессирующую гипонатриемию.

Все пациенты получали адекватную медикаментозную терапию в соответствии с существующими стандартами лечения ХCH, ишемической болезни сердца, артериальной гипертензии $[12,13]$ и соблюдали водно-питьевой и солевой режим [2]. В период активной диуретической терапии пациентам рекомендовали умеренное ограничение натрия с пищей (< 3 г/сут), после достижения компенсации состояния - согласно функциональному классу (ФК) ХСН. При
XСН I ФК пациентам рекомендовали не употреблять соленую пищу (ограничение натрия до 3 г/ сут), при II ФК - не подсаливать пищу (ограничение натрия до 1,5-2 г/сут), при III-IV ФК- использовать продукты с пониженным содержанием соли и готовить блюда без соли (ограничение натрия до 1 г/сут). Во время активного лечения диуретиками больным предписывали ограничение потребления жидкости до 1,5 л/сут, после достижения полной компенсации ХCH - менее 2 л/сут.

Обработку результатов выполняли на персональном компьютере с использованием пакета прикладных статистических программ «MedStat». Для проверки распределения на нормальность применяли критерий W ШапироУилки. При нормальном распределении количественные признаки были представлены в виде среднее \pm стандартное отклонение $(\mathrm{m} \pm \sigma)$, при отличном от нормального - обозначены как медиана и 1-й, 3-й квартили (Me (Q1; Q3)). Для сравнения двух выборок непрерывных переменных, подлежащих нормальному закону распределения, использовали t-критерия Стьюдента, при отличном от нормального - критерий Вилкоксона. Для сравнения трех выборок, не подчиняющихся нормальному закону распределения, проводился ранговый однофакторный дисперсионный анализ Крускала-Уоллиса, и при наличии статистически значимых различий между группами выполнялось сравнение с использованием критерия Данна. Для изучения распределения дискретных признаков в разных группах и сравнения относительных величин применяли стандартный метод анализа таблиц сопряженности с использованием критерия $\chi 2$. Во всех случаях проверки гипотез различия считались статистически значимыми при величине $\mathrm{p}$ $<0,05$.

\section{РЕЗУЛЬТАТЫ И ОБСУЖДЕНИЕ}

Анализ показал, что в целом у 132 (33,3 \%; 95\% доверительный интервал (ДИ) 28,8-38,0\%) из 396 пациентов была обнаружена гипонатриемия, у остальных 264 (66,7\%, 95\% ДИ 62,0-71,2\%) концентрация натрия крови находилась в пределах нормы. Медиана уровня сывороточного натрия у пациентов с гипонатриемией составила $132,5(131,0 ; 133,5)$ ммоль/л, у лиц с нормонатриемией - 139,5 (138,0; 142,5) ммоль/л.

Абсолютное большинство пациентов (112 (84,8\%; 95\% ДИ 78,2-90,5\%)) имели гипонатриемию легкой степени, у 14 (10,6\%; 95\% ДИ 5,9$16,5 \%)$ больных была обнаружена гипонатриемия умеренной степени и лишь у 6 (4,5\%; 95\% ДИ 1,6-8,8\%) - тяжелой.

Подробно была проанализирована распро- 
страненность различных типов гипонатриемии в зависимости от сроков её возникновения. Догоспитальная гипонатриемия (сниженный уровень натрия крови при поступлении в стаци- онар) была выявлена у 64 (48,5\%; 95\% ДИ 39,9$57,1 \%)$ пациентов, в то время как у остальных 68 (51,5\%, 95\% ДИ 42,9-60,1\%) она развилась во время пребывания в стационаре.

Исходная клиническая характеристика пациентов с догоспитальной гипонатриемией,

Таблица 1. госпитальной гипонатриемией и нормальным уровнем натрия

\begin{tabular}{|c|c|c|c|}
\hline Параметр & $\begin{array}{c}\text { Догоспитальная } \\
\text { гипонатриемия } \\
(\mathrm{n}=64) \\
\end{array}$ & $\begin{array}{c}\text { Госпитальная } \\
\text { гипонатриемия } \\
(\mathrm{n}=68) \\
\end{array}$ & $\begin{array}{c}\text { Нормонатриемия } \\
(\mathrm{n}=264)\end{array}$ \\
\hline Возраст, годы, Ме (Q1; Q3) & $69(65 ; 73,5)^{*}$ & $70(66 ; 74,5)^{*}$ & $64(61 ; 66,5)$ \\
\hline Мужской пол, число больных (\%) & $38(59,4 \%)$ & $49(72,1 \%)$ & $157(59,5 \%)$ \\
\hline ИМТ, кг $/ \mathrm{M}^{2}, \mathrm{~m} \pm \sigma$ & $31,4 \pm 2,9$ & $30,8 \pm 3,1$ & $29,6 \pm 2,8$ \\
\hline САД, $\mathrm{mmHg}, \mathrm{m}^{ \pm} \sigma$ & $119,3 \pm 3,6^{*}$ & $118,2 \pm 2,9 *$ & $132,3 \pm 3,7$ \\
\hline ДАД, $\mathrm{mmHg}, \mathrm{m} \pm \sigma$ & $74,2 \pm 3,1$ & $70,6 \pm 1,9 *$ & $76,2 \pm 3,9$ \\
\hline ЧСС в покое, уд/мин, Ме (Q1; Q3) & $88(80 ; 94)$ & $85(79 ; 93)$ & $86(78 ; 96)$ \\
\hline \multicolumn{4}{|l|}{ Коморбидные состояния: } \\
\hline Артериальная гипертензия, число больных (\%) & $56(87,5 \%)$ & $62(91,2 \%)$ & $236(89,4 \%)$ \\
\hline $\begin{array}{l}\text { Инфаркт миокарда в анамнезе, } \\
\text { число больных (\%) }\end{array}$ & $48(75,0 \%)$ & $49(72,1 \%)$ & $164(62,1 \%)$ \\
\hline Фибрилляция предсердий, число больных (\%) & $19(29,7 \%)$ & $21(30,9 \%)$ & $49(18,6 \%)$ \\
\hline Инсульт в анамнезе, число больных (\%) & $8(12,5 \%)$ & $9(13,2 \%)$ & $31(11,7 \%)$ \\
\hline $\begin{array}{l}\text { Хроническая обструктивная болезнь легких, } \\
\text { число больных (\%) }\end{array}$ & $21(32,8 \%)^{*}$ & $28(41,2 \%)^{*}$ & $41(15,5 \%)$ \\
\hline Сахарный диабет 2-го типа, число больных (\%) & $24(37,5 \%)^{*}$ & $24(35,3 \%)^{*}$ & $49(18,6 \%)$ \\
\hline Анемия, число больных (\%) & $17(26,6 \%)^{*}$ & $19(27,9 \%)^{*}$ & $40(15,2 \%)$ \\
\hline \multicolumn{4}{|l|}{ Тяжесть ХСН: } \\
\hline ФК по NYHА, Mе (Q1; Q3) & IV (III; IV)* & IV (III; IV)* & III (III; IV) \\
\hline $\begin{array}{l}\text { Длительность анамне- } \\
\text { за ХCH, годы, Мe (Q1; Q3) }\end{array}$ & $7,5(5 ; 9)$ & $6(4,5 ; 9)$ & $6(3,5 ; 9,5)$ \\
\hline $\begin{array}{l}\text { Признаки застоя по двум кругам } \\
\text { кровообращения, число больных (\%) }\end{array}$ & $56(87,5 \%)^{*}$ & $61(89,7 \%)^{*}$ & $183(69,3 \%)$ \\
\hline Анасарка, число больных (\%) & $13(20,3 \%)$ & $17(25,0 \%)$ & $29(11,0 \%)$ \\
\hline $\begin{array}{l}\text { Дистанция теста с 6-минутной ходьбой } \\
(\mathrm{n}=286), \mathrm{m} \pm \sigma\end{array}$ & $154,5 \pm 9,1^{*}$ & $168,4^{ \pm} 11,3^{*}$ & $205,6 \pm 13,2$ \\
\hline ШОКС, баллы, Мe (Q1; Q3) & $9(8 ; 10)^{*}$ & $10(8 ; 11)^{*}$ & $7(6 ; 8)$ \\
\hline \multicolumn{4}{|l|}{ Параметры ЭхоКГ: } \\
\hline ФВ ЛЖ, $\%, \mathrm{~m}^{ \pm} \sigma$ & $41,6 \pm 6,9 *$ & $43,8 \pm 7,1^{*}$ & $49,6 \pm 7,8$ \\
\hline $\mathrm{E} / \mathrm{e}, \mathrm{m}^{ \pm} \sigma$ & $17,8 \pm 6,8^{*}$ & $18,4 \pm 7,9^{*}$ & $15,6 \pm 5,3$ \\
\hline \multicolumn{4}{|l|}{ Лабораторные показатели: } \\
\hline $\begin{array}{l}\text { Уровень натрия крови при поступлении, } \\
\text { ммоль/л, Me (Q1; Q3) }\end{array}$ & $132,5(132 ; 133,5)^{*}$ & $136,5(136 ; 137,5)^{*}$ & $139,5(138 ; 142,5)$ \\
\hline Гемоглобин крови, г/л, $\mathrm{m}^{ \pm} \sigma$ & $110,3 \pm 7,4^{*}$ & $108,4 \pm 9,4^{*}$ & $118,3 \pm 7,5$ \\
\hline Общий ХС крови, ммоль/л, m$\sigma$ & $4,9 \pm 0,6$ & $4,9 \pm 0,7$ & $4,8 \pm 0,6$ \\
\hline Общий белок крови, ммоль/л, m相 & $65,3 \pm 5,3$ & $67,2 \pm 5,4$ & $66,8 \pm 6,5$ \\
\hline СКФ, мл/мин, $\mathrm{m}^{ \pm} \sigma$ & $42,3 \pm 7,4^{*}$ & $44,8 \pm 6,4^{*}$ & $58,3 \pm 6,5$ \\
\hline Калий крови, ммоль/л, Ме (Q1; Q3) & $4,32(3,74 ; 5,02)$ & $4,10(3,76 ; 4,38) *$ & $4,42(3,83 ; 5,22)$ \\
\hline Хлор крови, ммоль/л, Ме (Q1; Q3) & $96,4(94,3 ; 98,5)^{*}$ & $95,7(93,9 ; 98,5)^{*}$ & $101,4(98,7 ; 102,4)$ \\
\hline Кальций крови, ммоль/л, Ме (Q1; Q3) & $1,11(0,99 ; 1,15)^{*}$ & $1,10(0,98 ; 1,13)^{*}$ & $1,19(1,13 ; 1,24)$ \\
\hline
\end{tabular}

Примечание: ДАД - диастолическое артериальное давление; ИМТ - индекс массы тела; САД - систолическое артериальное давление; СКФ - скорость клубочковой фильтрации; ФВ ЛЖ - фракция выброса левого желудочка; ФК - функциональный класс, ЧСС - частота сердечных сокращений; ХС - холестерин; ХСН - хроническая сердечная недостаточность; ФК - функциональный класс; ЭхоКГ - эхокардиография; * - различия статистически значимы (p<0,05) по сравнению с пациентами с нормонатриемией. 
Медиана уровня натрия в сыворотке крови у пациентов с догоспитальной гипонатриемией составила $132,5(132 ; 133,5)$ ммоль/л, а в группе с госпитальной гипонатриемией 132 (131; 133) ммоль/л, при этом различия не были статистически значимыми $(\mathrm{p}=0,093)$. В среднем госпитальная гипонатриемия развивалась на 6-е сутки пребывания в стационаре: медиана сроков возникновения составила $6,0(3,0 ; 6,0)$ дней.

Среди больных с догоспитальной гипонатримией была проанализирована динамика уровня натрия в течение периода стационарного лечения. Было установлено, что у 5 (7,8\%; 95\% ДИ 2,5-15,8\%) пациентов к моменту выписки произошла нормализация уровня натрия, у 16 (25,0\%; 95\% ДИ 15,1-36,5\%) - значимое ( 3 ммоль/л) прогрессирование гипонатриемии, у остальных 43 (67,2\%; 95\% ДИ 55,0-73,8\%) уровень натрия крови существенно не изменился.

Среди больных с госпитальной гипонатриемией лишь у 6 (8,8\%; 95\% ДИ 3,2-16,8\%) пациентов к моменту выписки произошла нормализация уровня натрия, у остальных 62 (91,2\%; 95\% ДИ 83,2-96,8\%) больных его концентрация оставалась ниже референтных значений.

Клиническая характеристика пациентов с гипо- и нормонатриемией представлена в таблице 1 .

Пациенты с гипонатриемией были достоверно ( $<0,05)$ старше по сравнению с лицами с нормальным уровнем натрия, среди них чаще встречались лица, имеющие анемию, сопутствующую хроническую обструктивную болезнь легких, сахарный диабет 2-го типа и нарушение функции почек. Значимых различий по индексу массы тела, распространенности артериальной гипертензии, фибрилляции предсер- дий, инфаркта миокарда между группами выявлено не было.

Больные с гипонатриемией имели более выраженные клинические признаки ХCH и ниже уровень артериального давления при поступлении. По данным эхокардиографического исследования они имели меньшую фракцию выброса и более выраженную диастолическую дисфункцию левого желудочка по сравнению с нормонатриемичными пациентами.

Согласно результатам лабораторного исследования у пациентов с гипонатриемией по сравнению с лицами с нормальным уровнем натрия были ниже уровни гемоглобина, скорости клубочковой фильтрации, ионизированного кальция и хлора крови, а у больных с госпитальной гипонатриемией, кроме того, ниже уровень калия крови.

При анализе догоспитальной медикаментозной терапии также был выявлен ряд различий: пациенты с гипонатриемией реже получали патогенетическую терапию ХСН и петлевые диуретики, и напротив, чаще принимали тиазидные и тиазидоподобные диуретики (табл. 2.).

Анализ водно-солевого режима пациентов в течение месяца, предшествующего госпитализации, показал, что лица с гипонатриемией чаще ограничивали потребление соли с пищей, а пациенты с госпитальной гипонатриемией при этом потребляли большие объемы жидкости по сравнению с нормонатриемичными больными (табл. 3.).

Анализ медикаментозной терапии, получаемой во время пребывания в стационаре, показал, что пациенты с гипонатриемией чаще получали тиазидные и тиазидоподобные диуретики по сравнению с больными с нормонатри-

Таблица 2.

Догоспитальная медикаментозная терапия в группах на момент поступления в стационар (число пациентов, \%)

\begin{tabular}{lccc}
\hline \multicolumn{1}{c}{ Группа препаратов } & $\begin{array}{c}\text { Догоспитальная } \\
\text { гипонатриемия }(\mathrm{n}=64)\end{array}$ & $\begin{array}{c}\text { Госпитальная } \\
\text { гипонатриемия }(\mathrm{n}=68)\end{array}$ & $\begin{array}{c}\text { Нормонатриемия } \\
(\mathrm{n}=264)\end{array}$ \\
\hline \hline Ингибиторы АПФ & $23(35,9 \%)^{*}$ & $19(29,7 \%)^{*}$ & $147(55,7 \%)$ \\
\hline АРА-2 & $3(4,7 \%)$ & $2(2,9 \%)$ & $18(6,8 \%)$ \\
\hline$\beta$-адреноблокаторы & $18(28,1 \%) *$ & $18(26,5 \%)^{*}$ & $126(47,7 \%)$ \\
\hline Статины & $12(18,8 \%)$ & $9(13,2 \%)$ & $54(20,5 \%)$ \\
\hline Петлевые диуретики & $7(10,9 \%)$ & $9(13,2 \%)$ & $84(31,8 \%)$ \\
\hline Антагонисты МКР & $7(10,9 \%)$ & $8(11,6 \%)$ & $69(26,1 \%)$ \\
\hline $\begin{array}{l}\text { Тиазидные и тиазидопо- } \\
\text { добные диуретики }\end{array}$ & $19(29,7 \%)^{*}$ & $5(7,4 \%)^{*}$ & $28(10,6 \%)$ \\
\hline
\end{tabular}

Примечание: АПФ - ангиотензинпревращающий фермент, АРА-2 - антагонисты рецепторов к ангиотензину-2, МКР - минералокортикоидные рецепторы; * - различия статистически значимы $($ р $<0,05)$ по сравнению с пациентами с нормонатриемией. 
емией. В группе с госпитальной гипонатриемией, кроме того, была выше частота назначения антагонистов минералокортикоидных рецепторов по сравнению с остальными группами больных (табл. 4.).

При детальном анализе диуретической терапии в период активной фазы были обнаружены различия и в дозах препаратов: пациентам с гипонатриемий назначались более высокие дозы гидрохлортиазида, индапамида и спиронолактона по сравнению с больными с нормальным уровнем натрия. Наибольшие дозы спиронолактона принимали больные с гипонатриемией, развившейся в период госпитализации (табл. 5.).
В настоящее время не остается сомнений, что гипонатриемия является наиболее частой электролитной аномалией среди пациентов, госпитализированных в стационар, вне зависимости от причины госпитализации. По данным крупного американского регистра, включающего 300 тыс. человек, частота гипонатриемии среди всех госпитализированных пациентов достигает 34\%, а её развитие ассоциируется с достоверным ухудшением исходов заболевания [14]. Гипонатриемия сопровождает течение многих заболеваний и состояний, таких как XCH, нефротический синдром, цирроз печени, онкологические и воспалительные болезни легких, неврологические расстройства [15]. Однако несмотря

Водно-солевой режим пациентов на догоспитальном этапе

Таблица 3. (число пациентов, \%)

\begin{tabular}{lccc}
\hline \multicolumn{1}{c}{ Группа препаратов } & $\begin{array}{c}\text { Догоспитальная } \\
\text { гипонатриемия }(\mathrm{n}=64)\end{array}$ & $\begin{array}{c}\text { Госпитальная } \\
\text { гипонатриемия (n=68) }\end{array}$ & $\begin{array}{c}\text { Нормонатриемия } \\
(\mathrm{n}=264)\end{array}$ \\
\hline \hline Резкое ограничение & \multicolumn{1}{c}{ Потребление соли } & $8(3 \%)$ \\
\hline Умеренное ограничение & $5(7,8 \%)$ & $10(14,7 \%)^{*}$ & $42(15,9 \%)$ \\
\hline Ограничение, всего & $26(40,6 \%)^{*}$ & $25(36,8 \%)^{*}$ & $50(18,9 \%)$ \\
\hline Нормальное потребление & $31(48,4 \%)^{*}$ & $35(51,5 \%)^{*}$ & $176(66,7 \%)$ \\
\hline Избыточное потребление & $24(37,5 \%)^{*}$ & $27(39,7 \%)^{*}$ & $38(14,4 \%)$ \\
\hline & $9(14,1 \%)$ & $6(8,8 \%)$ & $31(11,7 \%)$ \\
\hline$<1,0$ л & Количество потребляемой жидкости в сутки & $137(51,9 \%)$ \\
\hline $1,0-1,5$ л & $8(12,5 \%)$ & $11(16,2 \%)$ & $68(25,8 \%)$ \\
\hline $1,5-2,0$ л & $28(43,8 \%)$ & $17(25,0 \%)^{*}$ & $28(10,6 \%)$ \\
\hline$>2,0$ л & $19(29,7 \%)$ & $28(41,2 \%)^{*}$ & $12(17,6 \%)$ \\
\hline
\end{tabular}

Примечание: * - различия статистически значимы $(\mathrm{p}<0,05)$ по сравнению с пациентами с нормонатриемией.

Медикаментозная терапия во время пребывания в стационаре

Таблица 4 (число пациентов, \%)

\begin{tabular}{lccc}
\hline \multicolumn{1}{c}{ Группа препаратов } & $\begin{array}{c}\text { Догоспитальная } \\
\text { гипонатриемия (n=64) }\end{array}$ & $\begin{array}{c}\text { Госпитальная } \\
\text { гипонатриемия (n=68) }\end{array}$ & $\begin{array}{c}\text { Нормонатриемия } \\
(\mathrm{n}=264)\end{array}$ \\
\hline \hline Ингибиторы АПФ & $54(84,4 \%)$ & $53(77,9 \%)$ & $221(83,7 \%)$ \\
\hline АРА-2 & $10(15,6 \%)$ & $15(22,1 \%)$ & $38(14,4 \%)$ \\
\hline$\beta$-адреноблокаторы & $56(87,5 \%)$ & $59(86,8 \%)$ & $238(90,2 \%)$ \\
\hline Петлевые диуретики & $64(100,0 \%)$ & $68(100,0 \%)$ & $264(100,0 \%)$ \\
\hline Антагонисты МКР & $54(84,4 \%)$ & $66(97,1 \%) \#_{*}$ & $202(76,5 \%)$ \\
\hline $\begin{array}{l}\text { Тиазидные и тиазидо- } \\
\text { подобные диуретики }\end{array}$ & $19(29,7 \%)^{*}$ & $28(41,2 \%)^{*}$ & $(15,9 \%)$ \\
\hline
\end{tabular}

Примечание: АПФ - ангиотензинпревращающий фермент, АРА-2 - антагонисты рецепторов к ангиотензину-2, МКР - минералокортикоидные рецепторы; * различия статистически значимы $(p<0,05)$ по сравнению с пациентами с нормонатриемией; \# - различия статистически значимы $(\mathrm{p}<0,05)$ по сравнению с пациентами с догоспитальной гипонатриемией. 
Таблица 5.

Типы и среднесуточные дозы диуретиков в период активной диуретической терапии

\begin{tabular}{lccc}
\hline \multicolumn{1}{c}{ Группа препаратов } & $\begin{array}{c}\text { Догоспитальная } \\
\text { гипонатриемия }(\mathrm{n}=64)\end{array}$ & $\begin{array}{c}\text { Госпитальная } \\
\text { гипонатриемия }(\mathrm{n}=68)\end{array}$ & $\begin{array}{c}\text { Нормонатриемия } \\
(\mathrm{n}=264)\end{array}$ \\
\hline \hline \multicolumn{4}{l}{ Фетлевые диуретики: } \\
\hline $\begin{array}{l}\text { торосемид, число больных (\%) } \\
\begin{array}{l}\text { среднесуточная доза (в пересчете } \\
\text { на фуросемид), мг, (Ме (Q1; Q3)) }\end{array}\end{array} \quad 82(81,3 \%)$ & $46(67,7 \%)$ & $194(73,5 \%)$ \\
\hline
\end{tabular}

Тиазидные диуретики (гидрохлортиазид):

\begin{tabular}{lccc}
\hline число больных (\%) & $11(17,2 \%)$ & $27(39,7 \%)^{*}$ & $29(11,0 \%)$ \\
\hline $\begin{array}{l}\text { среднесуточная доза, } \\
\text { мг (Me (Q1; Q3)) }\end{array}$ & $25(12,5 ; 25)^{*}$ & $25(12,5 ; 25)^{*}$ & $12,5(12,5 ; 25)$ \\
\hline
\end{tabular}

Тиазидоподобные диуретики (индапамид):

\begin{tabular}{|c|c|c|c|}
\hline число больных (\%) & $8(12,5 \%)$ & $11(16,2 \%)^{*}$ & $13(4,9 \%)$ \\
\hline $\begin{array}{l}\text { среднесуточная доза, } \\
\text { мг (Mе (Q1; Q3)) }\end{array}$ & $1,25(1,25 ; 1,5)^{*}$ & $1,25(1,25 ; 1,5)^{*}$ & $0,625(0,625 ; 1,25)$ \\
\hline \multicolumn{4}{|c|}{ Антагонисты минералокортикоидных рецепторов: } \\
\hline $\begin{array}{l}\text { спиронолактон, чис- } \\
\text { ло больных (\%) }\end{array}$ & $51(79,7 \%)$ & $62(93,9 \%)^{*} \#$ & $188(71,2 \%)$ \\
\hline $\begin{array}{l}\text { спиронолактон, среднесуточ- } \\
\text { ная доза, мг (Ме (Q1; Q3)) }\end{array}$ & $100(75 ; 100)^{*}$ & $150(100 ; 150)^{*} \#$ & $75(50 ; 100)$ \\
\hline эплеренон, число больных (\%) & $3(4,7 \%)$ & $4(5,9 \%)$ & $14(5,3 \%)$ \\
\hline $\begin{array}{l}\text { эплеренон, среднесуточ- } \\
\text { ная доза, мг (Mе (Q1; Q3)) }\end{array}$ & $25(25 ; 25)$ & $25(25 ; 25)$ & $(25 ; 25)$ \\
\hline
\end{tabular}

Примечание: * - различия статистически значимы $(\mathrm{p}<0,05)$ по сравнению с пациентами с нормонатриемией; \# различия статистически значимы $(\mathrm{p}<0,05)$ по сравнению с пациентами с догоспитальной гипонатриемией.

на столь высокую распространенность и прогностическое значение гипонатриемии, осведомленность клиницистов о данной проблеме по-прежнему остается невысокой.

Снижение уровня натрия крови при ХCH является вариантом гиперволемической гипернатриемии и вызвано преимущественно нарушением выделения воды организмом [16]. При этом патофизиологической основой развития гипонатриемии в данном случае является избыточная нейрогуморальная активация в условиях снижения сердечного выброса. Несмотря на абсолютный избыток воды во внеклеточном пространстве организма при декомпенсации ХСН, эффективный объем циркулирующей крови остается низким, что через систему барорецепторов способствует неосмотической стимуляции секреции антидиуретического гормона и эффекторов ренин-ангиотензинальдостероновой системы. Такая нейрогуморальная активация носит компенсаторный характер и направлена на нормализацию перфузионного давления за счет ограничения экскреции натрия и воды. Выделение антидиуретического гормона непосредственно усиливает реабсорбцию воды в собирательных канальцах по- чек, тогда как ангиотензин-2 и норадреналин ограничивают доставку воды к почкам путем уменьшения их перфузии и следовательно, способствуют снижению её выделения. Кроме того, снижение сердечного выброса и высокий уровень ангиотензина-2 являются мощными стимулами жажды, что приводит к увеличению потребления воды [17].

Степень нейрогуморальной активации при $\mathrm{XCH}$, а следовательно, и риск развития гипонатриемии, как правило, коррелирует с тяжестью кардиальной дисфункции, что подтверждается в нашем исследовании. Действительно, пациенты с гипонатриемией имели более тяжелые клинические (функциональный класс по NYHA, выраженность застойных явлений, дистанция теста с 6-минутной ходьбой) и эхокардиографические (степень систолической и диастолической дисфункции) признаки ХСН по сравнению с пациентами с нормальным уровнем натрия крови. Кроме того, на догоспитальном этапе они реже получали медикаментозную терапию, направленную на подавление избыточной нейрогуморальной активации (ингибиторы ангиотензинпревращающего фермента, антагонисты рецепторов ангиотензина-2, $\beta$-адреноблокаторы). 
Нельзя исключить, что определенный вклад в развитие госпитальной гипонатриемии вносит и активная диуретическая терапия. Так, по результатам проведенного нами исследования, пациенты с гипонатриемией достоверно чаще и в более высоких дозах получали тиазидные, тиазидоподобные диуретики и спиронолактон. Примечательно, что наибольшие дозы спиронолактона принимали больные с гипонатриемией, развившейся в период госпитализации. Разумеется, обеспечение адекватного натрий- и диуреза является важнейшим фактором профилактики симптомов декомпенсации ХCH, а при развитии признаков гиперволемии - необходимым условием для их устранения. Тем не менее стоит помнить, что применение диуретической терапии может сопровождаться развитием нежелательных побочных эффектов, среди которых лидирующие позиции занимают различные электролитные нарушения, в том числе гипонатриемия. По данным литературы, наиболее часто гипонатриемия наблюдается при использовании тиазидных и тиазидоподобных диуретиков, реже - петлевых и калийсберегающих [18]. Тиазидные диуретики обладают смешанным механизмом гипонатриемического действия: они усиливают эффект антидиуретического гормона на уровне собирательных трубок и в то же время стимулируют натрийурез. Развитие гипонатриемии при применении спиронолактона, помимо натрийуретического эффекта препарата, связывают с его способностью блокировать выход натрия из клетки, что приводит к повышению внутриклеточного содержания электролита и уменьшению его концентрации в крови.

Таким образом, активная диуретическая терапия у больных с декомпенсцацией ХCH, в осо- бенности с применением тиазидных, тиазидоподобных диуретиков и высоких доз спиронолактона, следует проводить с осторожностью, под контролем уровня электролитов крови, что регламентируется современными руководствами [19].

Остается неясным, является ли гипонатреимия per se фактором, определяющим ухудшение прогноза при ХСН, либо она лишь выступает лабораторным маркером тяжести заболевания. Для ответа на этот вопрос необходимо проведение дальнейших крупных исследований. Тем не менее, в любом случае, снижение уровня натрия крови может служить простым и надежным инструментом стратификации риска у больных с декомпенсацией ХCH.

\section{З АК ЛЮ ЧЕНИЕ}

Гипонатриемия является частым нарушением водно-электролитного баланса у пациентов, госпитализированных по поводу декомпенсации ХСН. В целом её распространенность достигает 33,3 \%, при этом у 48,5\% больных она развивается на догоспитальном этапе, а у 51,5\% (95\% ДИ 42,9-60,1\%) - во время лечения в отделении. Госпитальная гипонатриемия развивается в среднем через $6(3 ; 6)$ дней лечения.

В превалирующем большинстве случаев у пациентов с декомпенсацией ХCH развивается гипонатриемия легкой степени, в то время как частота умеренной и тяжелой гипонатриемии является невысокой $(84,8 \%$ и 4,6\% соответственно).

К моменту выписки спонтанная нормализация уровня натрия происходит лишь у 7,8\% больных, у 25\% пациентов, напротив, наблюдается прогрессирование гипонатриемии.

\section{Н.Т. Ватутин ${ }^{1,2}$, А.Н. Шевелёк ${ }^{1,2}$, А.Н. Загоруйко ${ }^{1}$}

${ }^{1}$ ГОО ВПО «Донецкий национальный медицинский университет имени М. Горького», Донецк

${ }^{2}$ Институт неотложной и восстановительной хирургии им. В.К. Гусака, Донецк

\section{РАСПРОСТРАНЕННОСТЬ ДОГОСПИТАЛЬНОЙ И ГОСПИТАЛЬНОЙ ГИПОНАТРИЕМИИ У БОЛЬНЫХ С ДЕКОМПЕНСАЦИЕЙ ХРОНИЧЕСКОЙ СЕРДЕЧНОЙ НЕДОСТАТОЧНОСТИ}

Целью исследования стала оценка распространенности различных типов гипонатриемии при декомпенсации хронической сердечной недостаточности (ХCH). Проведено проспективное исследование с участием 396 пациентов, госпитализированных по поводу декомпенсации ХСН. Установлено, что распространенность гипонатриемии (уровень натрия крови < 135 ммоль/л) достигает 33,3 \%, при этом у 48,5\% больных она развивается на догоспитальном этапе, а у 51,5\% во время лечения в отделении. Госпитальная гипонатриемия развивается в среднем через $6(3 ; 6)$ дней лечения. В превалирующем большинстве случаев у па- циентов с декомпенсацией ХСН развивается гипонатриемия легкой степени, в то время как частота умеренной и тяжелой гипонатриемии является невысокой (84,8\% и 4,6\% соответственно). К моменту выписки спонтанная нормализация уровня натрия происходит лишь у 7,8\% больных, у 25\% пациентов, напротив, наблюдается прогрессирование гипонатриемии.

Ключевые слова: госпитальная гипонатриемия, догоспитальная гипонатриемия, натрий крови, хроническая сердечная недостаточность, декомпенсация. 


\section{N.T. Vatutin ${ }^{1,2}$, A.N. Shevelyok ${ }^{1,2}$, A.N. Zagoruiko ${ }^{1}$}

${ }^{1}$ SEI HPE «M. Gorky Donetsk National Medical University», Donetsk ${ }^{2}$ V.K. Gusak Institute of Urgent and Reconstructive Surgery, Donetsk

\section{PREVALENCE OF PREHOSPITAL AND HOSPITAL HYPONATREMIA IN PATIENTS WITH DECOMPENSATED CHRONIC HEART FAILURE}

The aim of the study was to assess the prevalence of different types of hyponatremia in decompensation of chronic heart failure (CHF). A prospective study included 396 patients hospitalized for decompensated CHF. The prevalence of hyponatremia (blood sodium level $<135$ $\mathrm{mmol} / \mathrm{l}$ ) was $33.3 \%$. In $48.5 \%$ cases it developed before admission (prehospital hyponatremia), while in $51,5 \%-$ during hospitalization (hospital hyponatremia). Hospital hyponatremia developed on average $6(3 ; 6)$ days of treatment. Prevailing majority of patients had mild hyponatremia. The incidence of moderate and severe hyponatremia was low ( $84.8 \%$ and $4.6 \%$, respectively). At the time of discharge spontaneous normalization of the sodium level occurs in only $7.8 \%$ of patients, while in $25 \%$ of patients there was progression of hyponatremia.

Key words: hospital hyponatremia, prehospital hyponatremia, blood sodium, chronic heart failure, decompensation.

\section{ЛИТЕРАТУРА}

1. Montalescot G., Sechtem U., Achenbach S. 2013 ESC guidelines on the management of stable coronary artery disease. European Heart Journal. 2013; 34: 2949-3003.

2. Мареев В.Ю., Агеев Ф.Т., Арутюнов Г.П. Национальные рекомендации ОССН, РКО и РНМОТ по диагностике и лечению ХСН (четвертый пересмотр). Журнал Сердечная Недостаточность. 2013; 14 (7).

3. Filippatos T.D., Elisaf M.S. Hyponatremia in patients with heart failure. World J Cardiol. 2013; 5 (9): 317-328.

4. Щекочихин Д.Ю., Копылов Ф.Ю., Козловская Н.Л. Рациональная фармакотерапия в кардиологии. 2014; 10 (6): $1-2$.

5. SpasovskiG., VanholderR., AllolioB., etal. Clinical practice guideline on diagnosis and treatment of hyponatraemia. Nephrol Dial Transplant. 2014; 40 (6): 924.

6. Gheorghiade M. Characterization and prognostic value of persistent hyponatremia in patients with severe heart failure in the ESCAPE Trial. Arch Intern Med. 2007; 167 (18): 1998-2005.

7. Filippatos G., Rossi J., Lloyd-Jones D.M. et al. Prognostic value of blood urea nitrogen in patients hospitalized with worsening heart failure: insights from the Acute and Chronic Therapeutic Impact of a Vasopressin Antagonist in Chronic Heart Failure (ACTIV in CHF) study. J Card Fail. 2007; 13: 360-364.

8. Romanovsky A., Bagshaw S., Mitchell H. Hyponatremia and Congestive Heart Failure: A Marker of Increased Mortality and a Target for Therapy. Int J Nephrol. Rosner. 2011.

9. Ponikowski P., Voors A.A., Anker D.S. et al. 2016 ESC Guidelines for the diagnosis and treatment of acute and chronic heart failure: The Task Force for the diagnosis and treatment of acute and chronic heart failure of the European Society of Cardiology (ESC). EHJ. 2016; 37 (27): 2129-2200.

10. Hillier T.A., Abbott R.D., Barrett E.J. Hyponatremia: evaluating the correction factor for hyperglycemia. Am J Med. 1999; 106 (4): 399-403.

11. Кишкун А. А. Клиническая лабораторная диагностика: учебное пособие. 2015; 458.

12. Decourcelle A. Influence of previous physical activity on the outcome of patients treated by thrombolytic therapy for stroke. J. Neurol. 2015; 262 (11): 2513-2519.

13. Fraisl P. Inhibition of oxygen sensors as a therapeutic strategy for ischaemic and inflammatory disease. Nat. Rev. Drug. Discov. 2009; 8: 139-152.

\section{REFERENCES}

1. Montalescot G., Sechtem U., Achenbach S. 2013 ESC guidelines on the management of stable coronary artery disease. European Heart Journal. 2013; 34: 2949-3003.

2. Mareev V.Yu., Ageev F.T., Arutyunov G.P. Natsionalnyie rekomendatsii OSSN, RKO i RNMOT po diagnostike i lecheniyu HSN (chetvertyiy peresmotr). Zhurnal Serdechnaya Nedostatochnost [National recommendations of OSSN, RKO and RNMOT on diagnosis and treatment of CHF (fourth revision). Journal of Heart Failure]. 2013; 14 (7) (In Russian).

3. Filippatos T.D., Elisaf M.S. Hyponatremia in patients with heart failure. World J Cardiol. 2013; 5 (9): 317-328.

4. Schekochihin D.Yu., Kopyilov F.Yu., Kozlovskaya N.L. Ratsionalnaya farmakoterapiya v kardiologii [Rational pharmacotherapy in cardiology]. 2014; 10 (6): 1-2 (In Russion).

5. Spasovski G., Vanholder R., Allolio B., et al. Clinical practice guideline on diagnosis and treatment of hyponatraemia. Nephrol Dial Transplant. 2014; 40 (6): 924.

6. Gheorghiade M. Characterization and prognostic value of persistent hyponatremia in patients with severe heart failure in the ESCAPE Trial. Arch Intern Med. 2007; 167 (18): 1998-2005.

7. Filippatos G., Rossi J., Lloyd-Jones D.M. et al. Prognostic value of blood urea nitrogen in patients hospitalized with worsening heart failure: insights from the Acute and Chronic Therapeutic Impact of a Vasopressin Antagonist in Chronic Heart Failure (ACTIV in CHF) study. J Card Fail. 2007; 13: 360-364.

8. Romanovsky A., Bagshaw S., Mitchell H. Hyponatremia and Congestive Heart Failure: A Marker of Increased Mortality and a Target for Therapy. Int J Nephrol. Rosner. 2011.

9. Ponikowski P., Voors A.A., Anker D.S. et al. 2016 ESC Guidelines for the diagnosis and treatment of acute and chronic heart failure: The Task Force for the diagnosis and treatment of acute and chronic heart failure of the European Society of Cardiology (ESC). EHJ. 2016; 37 (27): 21292200.

10. Hillier T.A., Abbott R.D., Barrett E.J. Hyponatremia: evaluating the correction factor for hyperglycemia. Am J Med. 1999; 106 (4): 399-403.

11. Kishkun A.A. Klinicheskaya laboratornaya diagnostika: uchebnoe posobie [Clinical laboratory diagnostics: a textbook]. 2015; 458 (In Russion).

12. Decourcelle A. Influence of previous physical activity on 
14. Hawkins R.C. Age and gender as risk factors for hyponatremia and hypernatremia. Clin Chim Acta 2003; 337: 169172.

15. Braun, Michael M., Barstow, Craig H., Pyzoha, Natasha J. Diagnosis and Management of Sodium Disorders: Hyponatremia and Hypernatremia. AAFP. 2015; 91 (5): 299-307.

16. VerbruggeF.H., Steels P., GrietenL., NijstP.Hyponatremia in Acute Decompensated Heart Failure Depletion Versus Dilution. JAAC. 2015; 65 (5): 481-486.

17. Adrogué H.J. Hyponatremia in Heart Failure. Cardiovasc J. $2017 ; 13$ (1): 40. the outcome of patients treated by thrombolytic therapy for stroke. J. Neurol. 2015; 262 (11): 2513-2519.

13. Fraisl P. Inhibition of oxygen sensors as a therapeutic strategy for ischaemic and inflammatory disease. Nat. Rev. Drug. Discov. 2009; 8: 139-152.

14. Hawkins R.C. Age and gender as risk factors for hyponatremia and hypernatremia. Clin Chim Acta 2003; 337: 169172.

15. Braun, Michael M., Barstow, Craig H., Pyzoha, Natasha J. Diagnosis and Management of Sodium Disorders: Hyponatremia and Hypernatremia. AAFP. 2015; 91 (5): 299-307.

16. Verbrugge F. H., Steels P., Grieten L., Nijst P. Hyponatremia in Acute Decompensated Heart Failure Depletion Versus Dilution. JAAC. 2015; 65 (5): 481-486.

17. Adrogué H.J. Hyponatremia in Heart Failure. Cardiovasc J. $2017 ; 13$ (1): 40 . 